\title{
Die Integrierte Versorgung per Gesetz ist für die Psychiatrie gescheitert
}

\author{
Integrated Care as Decreed by German Law Fails for Psychiatry
}

Bibliografie

Dol $10.1055 / \mathrm{s}-2007-970935$

Psychiat Prax 2008; 35: 57-59

(c) Georg Thieme Verlag KG

Stuttgart $\cdot$ New York.

ISSN 0303-4259

Korrespondenzadressen

Prof. Dr. med. Paul-Otto

Schmidt-Michel

Zentrum für Psychiatrie

Die Weissenau

Weingartshofer Straße 2

88214 Ravensburg

paul-otto.schmidt-michel@

zfp-weissenau.de

Dr. med. Frank Bergmann,

Facharzt für Neurologie,

Psychiatrie und

Psychotherapie

Theaterplatz 17

52062 Aachen

bergmann@bvdn-nordrhein.de

\section{Pro}

Die Finanzierung der integrierten Versorgung (IV) nach §140a SGB V basiert auf einem Budgetabzug bei den Leistungserbringern. Sie ermöglicht den gesetzlichen Krankenkassen (GKV) kommerzielle Vertragsfreiheit und gibt ihnen die Chance, ein wirtschaftlich und medizinisch effizientes Case-Management durch ökonomische Anreize für die Beteiligten zu gestalten. IVVerträge müssen entweder das Kriterium „fächerübergreifend" (im Sinne der Weiterbildungsordnung) oder „sektorübergreifend“ (ambulantstationär) erfüllen. Die IV könnte damit einige der anerkannten Übel des deutschen Gesundheitswesens überwinden: die Sektortrennung insbesondere zwischen dem stationären, ambulanten und rehabilitativen Bereich mit ihren Ressourcen raubenden medizinischen Doppelstrukturen sowie die fehlende Verzahnung fachübergreifender Behandlungspfade. Durch die integrierte GKV-Versorgung in der vorliegenden gesetzlichen Fassung beabsichtigt der Gesetzgeber, den Wettbewerb um Mitglieder unter den Kassen durch eine Verbesserung der Qualität der Versorgung zu fördern und durch eine Steuerung von Behandlungsprozessen Kostensenkung zu erreichen. Die Lage im 7. Jahr der IV ist jedoch unklar: Valide Erfahrungen und belastbare Daten zur Qualitätsentwicklung und Rentabilität stehen bislang aus. Immer wieder werden jedoch Zweifel an der Wirksamkeit und Sinnhaftigkeit laut [1-3].

Eine seit dem letzten Jahr regelmäßig aktualisierte Erhebung der DGPPN zum Stand der Integrierten Versorgung aus Sicht der Psychiatrie zeigt, dass die psychiatrisch-psychotherapeutischen Versorgungsanbieter bisher als Vertragspartner von IV-Verträgern nur bei wenigen Vertragsabschlüssen vertreten sind, obwohl ein Budgetabzug in gleicher Höhe wie in der somatischen Medizin erfolgt. Von 3309 gemeldeten IV-Verträgen (Stichtag 31.12.2006) [4] entfallen nur ca. 30 auf das psychiatrisch-psychotherapeutische Versorgungssystem (Stand Februar 2007) [5], ein Großteil der Verträge wurde im Versorgungsbereich der endoprothetischen Versorgung abgeschlossen. Die wenigen uns bekannten IV-Verträge im psychiatrisch-psychotherapeutischen Bereich haben folgende Charakteristika:

- drei Projekte (Hannover, Bremen, Mittweida) beinhalten IV-Verträge, in denen eine parallele Behandlung in einer Institutsambulanz z.T. explizit ausgeschlossen ist, obwohl die meisten zu betreuenden IV-Patienten in diesen Verträgen das Einschlusskriterium einer Institutsambulanzbehandlung aufweisen (Art, Dauer oder Schwere der Erkrankung). Vertragspartner sind niedergelassene Psychiater/ Nervenärzte und komplementäre Anbieter (psychiatrische Pflegedienste/Soziotherapieanbieter).

- Ein IV-Vertrag (Mainz) beinhaltet das Angebot einer begrenzten Zahl von psychotherapeutischen Gruppensitzungen (30) nach Überweisung durch Allgemeinärzte (37 Teilnehmer) hier sind die niedergelassenen Vertragspsychotherapeuten als potenzielle Anbieter ausgeschlossen.

- Ein IV-Vertrag (Aachen) konzentriert sich auf die Implementierung einer leitliniengestützten und an eigens entwickelten Behandlungspfaden ausgerichteten Behandlung von depressiven Patienten. Teilnehmer sind die Universitätsklinik, niedergelassene Fachärzte für Nervenheilkunde und Psychiatrie sowie Hausärzte. Neben der verbesserten Behandlungsqualität werben die Vertragspartner mit Vorteilen wie verkürzten Wartezeiten, intensiver Netzwerkbetreuung, strukturierter Psychoedukation und einem individuellen Erinnerungsservice.

- Ein IV-Vertrag (München) beschränkt sich auf das Angebot von Compliance-Schulungen für 
Patienten mit der Diagnose Schizophrenie und Depression und deren Angehörige.

- Weitere IV-Verträge sind Abschlüsse zwischen singulären Anbietern von Rehabilitationsleistungen (Reha-Kliniken) und Niedergelassenen mit kleinen Krankenkassen, von denen keine Impulse zur Struktur- und Prozessveränderung ausgehen. Hier ist das Hauptmotiv die Umgehung des gesamten spezialisierten psychiatrisch-psychotherapeutischen GKVKomplexes und die direkte Zuführung der Klienten in die Kostenträgerschaft des Rentenversicherungsträgers.

- Lediglich das Projekt in Itzehoe/Schleswig beinhaltet den sektorübergreifenden Gesamtkomplex stationärer, teilstationärer und ambulanter Behandlung (PIA) im Rahmen der Erprobung eines Globalbudgets mit dem Ziel der Umsteuerung der Behandlung in mehr ambulante und teilstationäre Versorgung.

Die IV-Verträge in Mainz und Aachen beinhalten das Kriterium „fächerübergreifend“ im Sinne der Weiterbildungsordnung (Facharzt für Allgemeinmedizin und Facharzt für Psychiatrie/ Psychotherapie/Psychosomatik). Die IV-Verträge in Hannover/ Bremen/Mittweida beziehen komplementäre GKV-Leistungen wie Soziotherapie und z.T. ambulante psychiatrische Pflege mit ein. Das Projekt in Itzehoe ist im eigentlichen Sinne kein IV-Vertrag zwischen verschiedenen Leistungsanbietern, da nur die Psychiatrische Klinik in Itzehoe Vertragspartner ist. Die Verschiebung und Neujustierung der Sektoren „ambulant“/,stationär“ im Rahmen der Klinik mit PIA ist jedoch das Kernziel dieses Modells. Da Institutsambulanzen und teil- und vollstationäre Angebote in den meisten Regionen wie in Itzehoe bei einem pflichtversorgenden Träger angesiedelt sind, hatte es bisher für (Fach-) Kliniken und Abteilungen für Psychiatrie und Psychotherapie keinen Sinn, IV-Verträge mit anderen Trägern abzuschließen, da die Hauptklientel dieser Kliniken weitgehend identisch ist mit dem Versorgungsauftrag der Institutsambulanzen, die sektorübergreifend als Nahtstelle zwischen stationärem und komplementären Bereich fungieren. Für potenzielle IV-Verträge mit anderen Trägern bleiben also nur die Patientengruppen, die nicht in der Institutsambulanz behandelt werden, d. h. dem Kriterium der Art, Dauer oder Schwere der Erkrankung nicht entsprechen. Sektorübergreifende IV-Verträge zu etablieren hieße hier, Verträge mit niedergelassenen Fachärzten für Psychiatrie/Psychotherapie/Psychosomatik abzuschließen. Diese kommen in der Regel nicht zustande, weil der Ressourcenbedarf im Einzelfall kaum prognostiziert werden kann, da die Varianz der Verläufe zu hoch ist (ganz abgesehen von der Behandlungsbereitschaft des Einzelnen, mit welchem Psychotherapeuten und welchem psychotherapeutischen Verfahren er sich auf einen Behandlungsprozess einlässt). Das Mainzer Modell stellt hier eine Ausnahme dar, indem es die psychotherapeutische Leistung auf maximal 30 Gruppensitzungen generell beschränkt. Nach dem Mainzer IV-Modell ist es faktisch der stationäre Bereich mit vorgeschalteter Institutsambulanz, der die ambulante Unter- und Fehlversorgung in der Psychiatrie/Psychotherapie ausgleichen soll und eine fachlich geleitete Steuerungsfunktion übernimmt. Bei den IV-Verträgen in der somatischen Medizin geht es neben effektiveren Behandlungsketten für die Patienten und der Gewinnung einer neuen Klientel in der Regel um eine bessere Konkurrenzstellung gegenüber anderen Anbietern. Dieser Aspekt könnte in der Psychiatrie im ambulanten Bereich und unter Umständen auch für konkurrierende stationäre Anbieter in vergleichsweise gut ausgestatteten Versorgungsregionen eine Rolle spielen. Die einzelnen Krankenkassen möchten ihre Mitglieder mittels IV-Verträgen gezielt ansprechen und werben. Dies setzt ein abwägendes Urteil und eine Form der Patientensouveränität seitens der Versicherten voraus, die in diesem Bereich aller Erfahrung nach nur zum Teil gegeben ist. Es werden sich nur wenig interessierte psychisch Kranke finden, die sich bei der jeweiligen Krankenkasse in den entsprechenden IV-Vertrag „einschreiben“ und es bedürfte dazu eines höheren finanziellen Anreizes, als lediglich die Einsparung der Praxisgebühr von 10,- /Quartal anzubieten, wie dies in einigen IV-Verträgen der Fall ist. Ein Teil der GKV-Versicherten ist von der integrierten Versorgung ausgeschlossen, weil er nicht zu einer der für den Wettbewerb der Kassen relevanten Mitgliedergruppen gehört. In diesem Zusammenhang müssen auch Einwände von Betroffenen-Verbänden ernst genommen werden, die etwa Einschränkungen bei der freien Arztwahl und eine einseitige Reduzierung der Versorgung auf die medikalisierte Behandlung befürchten.

Für die Kernklientel der pflichtversorgenden psychiatrisch-psychotherapeutischen Kliniken verläuft die wesentliche Sektorgrenze jedoch nicht im GKV-Bereich, sondern zwischen dem GKV-Bereich und den SGB-IX-, -XI- und -XII-Bereichen. Vernetzungen zwischen diesen Bereichen im Sinne von Behandlungsund Betreuungspfaden brächten wirkliche Verbesserungen für die Betroffenen. Gemeindepsychiatrischer Verbund (GPV)-Verträge und Hilfeplankonferenzen sind hier Erfolg versprechendere und in vielen Regionen bereits realisierte Ansätze - dort sind in der Regel auch die komplementären GKV-Bereiche wie psychiatrische Behandlungspflege und Soziotherapie integriert - sofern sie bisher realisiert wurden. Die bisherigen o.g. Beispiele integrierter Versorgung im psychiatrisch-psychotherapeutischen Bereich stellen gemessen an ihrem Anspruch also eher einen Rückschritt hinter das Ergebnis einer 30-jährigen Psychiatriereform dar und arbeiten diesem (selbst dynamischen) Prozess gemeindenaher Versorgungsnetzwerke eher entgegen. Richtungweisende Merkmale weisen lediglich jene (wenigen) Verträge auf, die einen fachübergreifenden Ansatz zur Anwendung bringen.

„Integrierte Versorgung“ als „sektorübergreifende“ Versorgung im psychiatrischen/psychotherapeutischen/psychosomatischen Bereich hieße mit Blick auf die genannte Kernklientel der pflichtversorgenden Kliniken, die GKV-Fixierung zu überwinden und einer anderen Logik zu folgen: die tendenzielle Aufhebung der Finanzierungslogik des „belegten Bettes“ bzw. die Anpassung des Behandlungsbedarfs in stationären, teilstationären und ambulanten Settings intermittierend je nach in der Regel fluktuierendem Bedarf des einzelnen Patienten. Bei aller Kritik sind Ansätze, die Leitlinienorientierung und eine bessere Behandlungsstrukturierung umsetzen, aus Patientensicht immer ein Fortschritt. Vertraglich fixierte Kooperationen über die Schwellen von Fachbereichen und Sektorgrenzen hinweg können in diesem Rahmen zweifellos auf vorhandene Versorgungsmängel reagieren. Sie sollten aber nicht als politisches Feigenblatt für weiterhin bestehende Strukturfehler des nach wie vor fragmentierten Versorgungssystems missbraucht werden.

Es bleibt Fakt, dass die Integrierte Versorgung ökonomisch gesehen zulasten der Psychiatrie geht: Dem psychiatrischen Versorgungssystem wird aktuell mehr Budget abgezogen als über IVVerträge zurückfließt. Um diese fragwürdige Quersubventionierung zu beenden, ist eine Abkoppelung der IV-Verträge von den IV-Verträgen im somatischen Bereich aus unserer Sicht ein nun notwendiger, unabdingbarer Entwicklungsschritt. Korrekturbedürftig wäre ebenso, dass die Krankenkassen verpflichtet werden, Inhalte und Qualität der IV-Verträge öffentlich zu machen, ebenso die verwendeten Finanzmittel. 


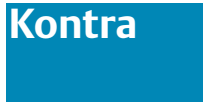

Es kann keine Rede davon sein, dass Integrierte Versorgung in der Psychiatrie gescheitert wäre. Im Gegenteil.

\section{Vorgeschichte der IV}

Gesundheitspolitiker aller Parteien hatten schon nach einem Gutachten des Sachverständigenrates zu Bedarfsgerechtigkeit und Wirtschaftlichkeit im Jahre 2002 Integrierte Versorgungsformen als das ideale Mittel für eine zielgerechte Verwendung der bekannten Ressourcen im Gesundheitssystem angesehen. Sie erhofften sich neben einer optimierten Behandlung auch Einsparmöglichkeiten durch bessere Definitionen der Schnittstellen. Jedoch erst durch finanzielle Anreize in Höhe von $1 \%$ der Gesamtvergütung kam es ab 2005 zu Bewegung in der gesundheitspolitischen Versorgungslandschaft, und es entstanden Integrierte Versorgungsmodelle. Ziel der Politik ist die Überwindung tradierter sektoraler Budgetierungen. Die Umstellung der Krankenhausfinanzierung auf DRG sowie die geplante Umstellung der vertragsärztlichen Honorierung auf morbiditätsorientierte Regelleistungsvolumina sind in diesem Zusammenhang wesentliche Eckpfeiler des Konzeptes.

\section{Startphase der IV}

Bemerkenswert ist, dass zu Beginn vor allem die Kostenträger mit der Vielzahl der eingereichten und vorgeschlagenen IV-Projekte augenscheinlich im Hinblick auf Kalkulation und Management überfordert waren. Darüber hinaus wurden die Projekte zunächst mit der apodiktischen Forderung Kostenersparnisse $\mathrm{zu}$ realisieren überfordert. Diese einseitige Betrachtungsweise führte in der Startphase zur Vernachlässigung von IV-Projekten ausgerechnet in Indikationen mit ausgewiesener Unterversorgung, wie beispielsweise in der Demenzversorgung oder in anderen psychiatrischen Indikationen. Darüber hinaus vermochten weder Kostenträger noch Gesundheitspolitiker lobbyistischen Versuchungen und Versprechungen zu widerstehen und stellten - mit Unterstützung des Gesundheitsministeriums - Integrierte Versorgung unter das Postulat der Hausarztzentrierung. Der daraus hervorgegangene Vertrag zwischen Hausarztverband und Barmer Ersatzkasse ist exemplarisch für einen Vertrag, der jegliche Zielvorgaben Integrierter Versorgung verfehlt.

Neben diesen eher kostenorientierten Projekten wie z.B. auch Verträgen zur Endoprothetik rücken jetzt zunehmend IV-Projekte mit ausgewiesener Qualitätsorientierung in den Vordergrund.

\section{Aktuelle Entwicklung der IV}

Als Beispiel kann das Integrierte Versorgungsmodell zur Versorgung depressiver Patienten dienen, welches als bundesweiter Rahmenvertrag zwischen den neuropsychiatrischen Berufsverbänden BVDN und BVDP mit der Techniker Krankenkasse abgeschlossen wurde und mittlerweile in vielen Regionen gestartet ist. Parallel wurde ein gleichlautender Vertrag mit der AOK Nordrhein geschlossen. Dieses IV-Modell wurde vor rund zwei Jahren in Aachen gestartet. Sowohl im Technikermodell als auch im AOK-Vertrag ist mittlerweile eine Indikationserweite- rung im Hinblick auf Versorgung von Patienten mit Psychosen vorgesehen. Professionelle Steuerung der Patienten im Netz, welches hausärztliche und fachärztliche ambulante und stationäre Versorgung integriert (unter Einbeziehung psychotherapeutischer und psychoedukativer Verfahren), zielorientiertes Case-Management, exakte Dokumentation sowie die Darstellung belastbarer Kennzahlen im Qualitätsmanagement machen es möglich, Patientengruppen mit unterschiedlichem Schweregrad der Erkrankung gezielt zu behandeln und definierte Zielgrößen zu erreichen, wie z.B. Verkürzung der Arbeitsunfähigkeitszeiten oder Absenkung der stationären Verweildauer. Dies gelingt mit flexiblem Ressourcenansatz innerhalb des Netzes. Mit anderen Worten: ausschließlich der Schweregrad der Erkrankung des Patienten steuert die Mittelverteilung im Netz. Weitere Verträge wurden mit Unterstützung der Berufsverbände in Nordrhein beispielsweise mit der DAK abgeschlossen, und zwar ein indikationsübergreifender Vertrag mit dem Ziel stationärer Einweisungen zu verhindern. Weitere Verträge sind auch in Berlin - ebenfalls indikationsübergreifend, aber auch in Niedersachsen, mit der DAK und anderen Kassen abgeschlossen worden. Die Homepage der DGPPN zählt aktuell 31 integrierte Versorgungsverträge in psychiatrischen Indikationen auf.

Aus Fehlern lernen: Integrierte Versorgung muss auch in der Regelversorgung problemlos durchführbar sein. Viele Dokumentationssysteme waren in der Startphase zu ambitioniert und umfangreich. Inzwischen wurden die Dokumentationen gestrafft und vereinheitlicht.

\section{Fazit}

Mittlerweile werden auf der Basis der beschriebenen Projekte zukunftsweisende IV-Strukturen in diversen psychiatrischen Indikationen entwickelt, die alle gleichen Regeln und Dokumentationssystemen folgen. Die wesentlichen Eckpfeiler sind:

- einfache Leistungsbeschreibung durch Basis- und Sonderkomplexe,

- Psychoedukation für Patienten und Angehörige im Sinne curriculär strukturierter Informationsveranstaltungen mit dem Ziel eines höheren Patienten-Empowerment durch verbesserten Informationsfluss und Förderung der Eigeninitiative und Eigenverantwortlichkeit,

- einheitliche Struktur- und Prozessqualität sowie Evaluation der Ergebnisqualität sowie

- schlanke Administration.

Nicht nur das Interesse der Kostenträger zeigt dabei, dass Integrierte Versorgung in der Psychiatrie gut aufgestellt ist.

\footnotetext{
Literatur

1 Kunze H, Priebe S. Integrierte Versorgung - Perspektiven für die Psychiatrie und Psychotherapie. Psychiat Prax 2006; 33: 353-355

2 Beske F, Ratschko K-W. Das GKV-Modernisierungsgesetz - GMG und seine Auswirkungen. Eine kritische Analyse. Kiel: 2006

3 Laschet H. Integration - Geschäft der Kassen auf Kosten von Kliniken und Ärzten. Ärztezeitung, 23.3.2007

4 Quelle http://www.bqs-register140d.de/

5 Quelle http://www.dgppn.de
} 